\title{
PSICOTERAPIA CENTRADA EN EL SENTIDO: "VIVIR CON SENTIDO". ESTUDIO PILOTO
}

\author{
MEANING-CENTERED GROUP PSYCHOTHERAPY: LIVE WITH SENSE. A PILOT STUDY
}

Francisco Luís Gil Moncayo' y William Breitbart².

' Unidad de Psico-Oncología. Institut Català d'Oncología. Hospital Duran i Reynals, Barcelona, Spain.

2 Memorial Sloan-Kettering Cancer Center, New York, NY, USA.

Resumen

Introducción: Los pacientes con cáncer avanzado expresan sentimientos de desesperanza, deseo de adelantar la muerte, y niveles elevados de ansiedad y sintomatología depresiva asociada a la pérdida de sentido de vida.

Objetivos: El principal objetivo de la Psicoterapia de Grupo Centrada en el Sentido (PGCS) es ayudar a los pacientes con cáncer a vivir con sentido, de una manera plena, manteniendo y ampliando el sentido de su vida, viviendo en paz y con un objetivo en la vida.

Participantes y Método: Actualmente, estamos realizando el estudio piloto, como primer paso del proyecto de validación del Modelo de Psicoterapia Centrada en el Sentido. Hemos realizado dos de los tres grupos de pacientes con cáncer avanzado que corresponde al presente estudio piloto, y estamos realizando las trascripción de cada una de la sesiones de los grupos, para su posterior análisis cualitativo.

Resultados: Los grupos de psicoterapia centrada en el sentido muestran una reducción de la sintomatología ansiosa y depresiva, desesperanza y deseo de adelantar la muerte en pacientes con cáncer avanzado, a la vez de aumentar el bienestar espiritual y la sensación de sentido.

Palabras claves: Terapia centrada en el sentido, terapia de grupo, cáncer, psicoterapia.

\section{Abstract}

Introduction: The patients with advanced cancer express feelings of hopelessness, wish to advance the death, and high levels of anxiety and depression associated to the loss of sense of life.

Aims: The main aim of the MeaningCentered Group Psychotherapy is to help to the patients with cancer to live with sense, of a full way, keeping and expanding the sense of his life, living in peace and with an aim in the life.

Participants and Method: At present, we are conducting the pilot study, as first step of the project of validation of the MeaningCentered Group Psychotherapy Model. We have performance two of the three groups of patients with advanced cancer that corresponds to the present pilot study, and are doing the transcription of each one of the sessions of the groups, for his back qualitative analysis.

Results: The Meaning-Centered Group Psychotherapy show a reduction of anxiety and depression, hopelessness and wish to advance the death in patients with advanced cancer, at the same time to increase the spiritual welfare and the feeling of sense.

Key words: Meaning-Centered Group Psychotherapy, group therapy, cancer, psychotherapy.

\section{Correspondencia:}




\section{INTRODUCCIÓN}

Un diagnóstico de cáncer puede ser una enfermedad con intención curativa o paliativa, pero siempre es una enfermedad tratable.

Los pacientes diagnosticados de cáncer avanzado (Estadios III y IV) presentan un mayor grado de complejidad, en relación al manejo de síntomas físicos y psicológi$\cos ^{(1,2,3)}$. Por ello, requieren de un modelo de intervención ajustado a sus necesidades. El modelo de psicoterapia desarrollado por William Breitbart ${ }^{(4)}$ ha demostrado su eficacia en este grupo de pacientes con cáncer avanzado.

\section{ANTECEDENTES Y ESTADO ACTUAL DEL TEMA}

Un diagnóstico de cáncer supone sentimientos de amenaza e incertidumbre para el paciente y la familia. En los estadios III y IV de enfermedades como el cáncer, el objetivo terapéutico es aumentar la supervivencia del paciente. La literatura nos muestra cómo dar sentido es importante para el paciente con cáncer: 1. focalizar la atención del paciente en que su vida tiene siempre sentido favorece el ajuste emocional a la enfermedad, 2. además de la alta prevalencia de distrés emocional observada, un número elevado de pacientes necesita dar sentido a su vida, a pesar de los síntomas físicos atribuibles a la enfermedad o tratamientos oncológicos, 3. los pacientes que experimentan que su vida tiene sentido, además de tener un mejor ajuste emocional a la enfermedad, su nivel de calidad de vida y confort es mayor ${ }^{(5)}$. Existe poca evidencia de cómo una intervención psicológica ayuda al paciente con cáncer a vivir plenamente y con sentido. El objetivo de este modelo de psicoterapia es estimular al paciente a la búsqueda de sentido, a través de experiencias, actitudes, revisión vital y desarrollo de su propio legado hacia sus fa- miliares. Consiste en ejercicios didácticos, experienciales y de discusión en grupo. Asimismo, tiene el objetivo de ayudar a los pacientes a "vivir con sentido", a pesar de las limitaciones físicas de la enfermedad, y los sentimientos de amenaza e incertidumbre que acompaña a la enfermedad. Los objetivos concretos son: búsqueda de sentido, reengancharse a la vida, capacidad para cambiar y para aceptar aquello que no podemos cambiar, normalizar e integrar la experiencia de tener cáncer, expresión emocional y apoyo mutuo, y mejorar el funcionamiento psicológico.

\section{PROCESOS, TÉCNICAS Y TAREAS}

\section{Descripción de la intervención}

El principal objetivo de la Psicoterapia de Grupo Centrada en el Sentido (PGCS) es ayudar a los pacientes con cáncer a vivir con sentido, de una manera plena, manteniendo y ampliando el sentido de su vida, viviendo en paz y con un objetivo en la vida. Más concretamente, los objetivos de la PGCS son: 1) favorecer que los pacientes con cáncer busquen sentido a su vida, a pesar de las incertidumbres y limitaciones de la enfermedad: a. reordenando/evaluando antiguos momentos con sentido, b. buscando nuevos sentidos a la vida, c. buscando formas de superar limitaciones prácticas; 2 ) buscar nuevas formas de reengancharse a la vida: trascender; 3) aprender a distinguir entre las limitaciones que podemos cambiar/superar, y aceptar aquellas que no se pueden cambiar; 4) integrar el diagnóstico de cáncer en la historia de vida del paciente; 5) expresar sentimientos y emociones; 6) mejorar la adaptación psicológica a través de darle sentido a la vida.

Cada sesión aborda un tema específico: introducción al concepto del sentido (1), cáncer y sentido (2), historia del concepto de sentido (3-4), fuentes del sentido (5-7), y cierre (8) (Cuadro 1). 


\section{Cuadro 1. Sesiones del Modelo de Psicoterapia Centrada en el Sentido}

1. La primera sesión_va dirigida a explicar el objetivo de la intervención psicoterapéutica individual centrada en el sentido (PICS). Ejercicio: el primer ejercicio experiencial va dirigido a que los pacientes narren momentos en que la vida fue particularmente significativa. Razón del ejercicio: el terapeuta explica como se desarrollará cada una de las sesiones, haciendo énfasis en el concepto central de las sesiones, mantener e incrementar el sentido de vida.

2. La sesión segunda aborda cómo el cáncer afecta el concepto de sentido de la persona. Ejercicio: responder a las siguientes dos preguntas: ¿Quién soy yo? ¿Cómo el diagnóstico de cáncer ha influido en la forma de verme? Razón del ejercicio: los pacientes pueden observar como el diagnóstico de cáncer ha podido cambiar el sentido de su vida.

3. La tercera y cuarta sesión aborda las fuentes pasadas, presentes y futuras que le darán sentido al paciente. Ejercicios: se realizan ejercicios autobiográficos, tanto durante la sesión, como en casa junto a su familia. Estos ejercicios tienen como objetivos crear una línea de vida, y valorar los objetivos significativos logrados a lo largo de la vida. Razón de los ejercicios: los pacientes realizan una labor de autoexploración, valorando cómo han abordado o manejado situaciones previas o cambios en su vida, y cuales han sido los logros u objetivos con significado que han logrado a lo largo de la vida.

4. En la quinta sesión, se le propone a los pacientes explorar o reflexionar a cerca de sus limitaciones presentes, principalmente por estar enfermo, y por la amenaza sobre la vida que conlleva la enfermedad. Ejercicios: las preguntas que se formulan son: ¿Puede hallar sentido a su vida a pesar de las limitaciones que debe afrontar a causa de su enfermedad?, ¿En alguna ocasión ha sentido que su vida ha perdido sentido?, ¿Qué es para usted morir en paz o con sentido? ¿Cómo le gustaría ser recordado? La ansiedad hacia la muerte será valorada cómo fuente de sentido. Razón de los ejercicios: favorecer que el paciente exprese sus emociones en relación con sus limitaciones y la amenaza de morir.

5. La sexta sesión se dirige a enfocar a los pacientes en qué acciones pueden realizar, desde un punto de vista de creatividad y responsabilidad. Ejercicios: se realizan las siguientes preguntas: ¿De qué es usted responsable?, ¿Qué cosas pendientes tiene aún por realizar?, ¿Qué cosas ha querido siempre realizar? Razón de los ejercicios: los pacientes son estimulados a explorar su capacidad de crear y de ser responsables de sus propias acciones en la vida.

6. La séptima sesión va dirigida a señalar hechos con sentido que hemos realizado en la vida. Ejercicios: se solicita a los pacientes recordar momentos de felicidad, de creatividad y de plenitud. Razón del ejercicio: los pacientes exploran el sentido de su vida a través de su experiencia.

7. La octava sesión va dirigida a que los pacientes puedan reflexionar y expresar planes y deseos para el futuro. Razón de los ejercicios: los pacientes pueden explorar qué emociones o sentimientos tienen en relación a sus limitaciones de vida.

8. Sesión a los dos meses de acabado el grupo. Va dirigida a evaluar el mantenimiento de la posible mejoría observada postratamiento de psicoterapia de grupo.

\section{INSTRUMENTOS DE EVALUACIÓN}

Los instrumentos de evaluación que utilizamos para valorar los cambios logrados a través de este modelo de psicoterapia están adaptados y validados en pacientes con cáncer en España. Son los siguientes:

1. Escala de Desesperanza ante la Enfermedad $^{(6)}$. Es una escala diseñada para 
evaluar el grado de desesperanza del enfermo. Consta de 8 ítems a los que el individuo ha de responder en una escala de 0 a 2.

2. Escala de Bienestar Espiritual (FACIT) $)^{(7)}$. Es una escala que evalúa la dimensión espiritual de la persona. Consta de 23 ítems, con 5 opciones de respuesta $(0$ nada en absoluto - 4 Mucho).

3. Adaptación Española del Test de Orientación Vital (Optimismo ${ }^{(8)}$. Es una escala que evalúa el grado de optimismo de la persona. Consta de 10 ítems, con cinco opciones de respuesta (0-4).

4. Escala de Depresión y Ansiedad Hospitalaria (HADS) $)^{(9,10)}$. Es una escala que evalúa la presencia de sintomatología ansiosa y depresiva. Consta de 14 ítems, 7 para sintomatología ansiosa y 7 para sintomatología depresiva. Puntuaciones mayores a 7 en cada una de las subescala de depresión o ansiedad se consideran como posibles casos clínicos.

5. Escala de Deseo de Adelantar la Muerte $(\mathrm{SAHD})^{(11)}$. Es una escala que evalúa el deseo del paciente de adelantar la muerte. Consta de 20 ítems a los que el individuo ha de responder verdadero o falso.

6. Escala de Crecimiento Postraumático $(\mathrm{PTGl})^{(12)}$. Evalúa el nivel de crecimiento personal o cambio en la vida como resultado de la enfermedad. Es una escala de 21 ítems con 5 subescalas: relación con los demás, nuevas posibilidades, fuerza personal, cambio espiritual y apreciación por la vida.

7. Escala de aburrimiento (PUB) ${ }^{(13)}$. Evalúa el nivel de aburrimiento, no estimulación y falta de propósito de vida en pacientes con cáncer. Consta de 14 ítems.

\section{ESTUDIO PILOTO}

Actualmente, hemos realizado dos de los tres grupos de 6-8 pacientes con cáncer avanzado que corresponde al presente estudio piloto, y estamos realizando las trascripción de cada una de la sesiones de los grupos, para su posterior análisis cualitativo.

El coordinador del estudio, y el segundo terapeuta (residente de Psicología Clínica) han dirigido las sesiones de los grupos, y han evaluado cada sesión, a través de las grabaciones audiovisuales realizadas, y han recogido los cuestionarios de evaluación rellenados antes de la primera sesión, tras la octava sesión de grupo, y a los dos meses acabado el mismo. Estas evaluaciones van dirigidas a mejorar el manual del terapeuta y el material de formación para próximos terapeutas que quieran formarse en este tipo de psicoterapia de grupo. A su vez, mejoraremos las técnicas y tareas que se utilizan para los próximos grupos, a través de una discusión activa entre los terapeutas expertos en grupo.

\section{CASO CLÍNICO}

Como caso clínico, incluimos la trascripción de la sesión 1 del primer grupo:

\section{Sesión 1}

\section{CONCEPTO Y FUENTES DE SENTIDO}

Pacientes: JA, ME, J, LU, CRIS, MONT. Falta: VAL

Terapeutas: Paco y Maribel

Paco: (Después de rellenar el primer cuestionario, antes de iniciar la primera sesión de grupo). Gracias por estar aquí a todos. Hoy es la primera sesión. Yo os conozco a todos, y vosotros no os conocéis entre vosotros. Como os comenté en la visita individual que tuvimos en consultas externas, el objetivo es compartir nuestras experiencias en grupo, con la finalidad de ayudar y sentirnos ayudados. Os he entregado una hoja, en cada sesión al inicio os entregaré una. Asimismo, os mandaré por e-mail un libro, "El hombre en busca del sentido" de Victor Frankl, para que os lo 
podáis comenzar a leer y espero que os guste. También sé que cuando nos dan un cuestionario, hay algunas preguntas que no nos gustan. Pero gracias a los cuestionarios, antes y después del grupo, sabremos si lo que hacemos aquí tiene alguna utilidad. Los cuestionarios comprenden preguntas sobre vuestro estado de ánimo, cómo afrontáis la situación que estáis viviendo. El grupo que comenzamos se Ilama "vivir con sentido", por que durante estas 8 sesiones vamos hablar mucho de sentido de vida, ¿Cuál es el propósito de mi vida, cuál es su sentido, y cómo el diagnóstico y el tratamiento están afectando el sentido de mi vida?. A mí me conocéis, me Ilamo Paco. Maribel (residente de Psicología), ¿Quieres presentarte?.

Maribel: Soy residente de Psicología, y estaré con vosotros en este grupo, ayudando a Paco y observando, y aprendiendo.

Paco: Muy bien, para comenzar, que cada uno de vosotros, en la medida que quiera compartir más o menos vuestra experiencia, que os presentéis al grupo, habléis de vuestra historia personal desde el diagnóstico hasta ahora. En este sentido, Javier si quieres comenzar.

J: Me llamo Javier, soy director financiero, en una empresa de publicidad, en relación a la enfermedad, me ha afectado, yo era una persona muy activa, y ahora me encuentro muy limitado por la actividad física que puedo hacer, y del tema que vamos a tratar del sentido, me afecta la posibilidad de la muerte.

Paco: Luisa...

LU: Me llamo Luisa, la enfermedad la llevo más o menos bien, ...me da todo igual...cuando el estado de ánimo me sube, siento como si no tuviera nada.

Paco: Montse.

MONT: Me llamo Montse, tengo 39 años, tengo 2 hijas, una de 8 y una de cuatro, cuando me diagnosticaron el cáncer, me hundí, pensé 39 años, dos hijas, tengo que salir hacia delante como sea (llora), y mi hija es la que me rompe el corazón, la que me da fuerza a mi, que dice que me van a curar, y yo saco fuerzas, y tengo ganas de vivir... mi marido lucha por mí, lleva mis hijas, Ileva la casa, lo lleva todo, y yo tengo ganas de vivir, es lo que le dije a la doctora, no te está funcionando la quimioterapia, y me están dando pastillas por que me operaron del riñón, me dijo la quimioterapia no te está funcionado, y yo le dije "yo no quiero morir", tengo ganas de vivir como sea, hemos empezado otra vez otra quimioterapia con pastillas a ver si me funciona, por que le dije que me diera tiempo, total que ahora estoy con estas pastillas hace 2 meses, mañana voy al médico, a ver como ha salido el TAC, yo me encuentro bien, cuando lo explico, mi forma de ser es llorar, sacar lo que yo siento, yo estoy luchando al cien por cien por mis hijas y por mi marido, ya está.

Paco: Gracias por tu sinceridad Montse. José Antonio

JA: Hola soy José, también tengo una hija de 15 años, trabajaba como técnico de laboratorio farmacéutico, y me diagnosticaron hace dos años también el tumor, el tipo de tumor no tiene curación, sí tiene tratamiento, y soy totalmente optimista, en cuanto a sobrevivir, conozco cómo funciona el tema de investigación y sé que tarde o temprano va a salir algo, para dentro de unos años esto será lo que antes era una gripe, que era una epidemia, sin embargo ahora aún nos falta para eliminar esto, pero lo que hay que hacer es sobrevivir aquí hasta que salga algo, y ya digo ser optimista en este aspecto, pero de todas las maneras, soy consciente de lo que tengo y tengo que asumir las cosas, ya no sé trata sólo de tengo que vivir como sea, sino que además intento disfrutar de cada día, tampoco puedo plantearme, soy consciente de lo que tengo, bueno, intento vivir al máximo cada momento y disfrutar de todo, cosas que antes no te pasaban por la cabeza, te la apuntas, ahora las disfrutas, 
preparas detalles que antes no preparabas, sobre todo a mí, sinceramente a pesar de todo, tengo que decir que esta es una experiencia positiva, en ese aspecto, a pesar de todo, claro, a pesar de la enfermedad

Paco: Sería cómo una experiencia de crecimiento personal.

JA: Sí, lo del tema personal, evidentemente, me genera mucho dolor muchas veces, y me cago en todo, y a veces, bueno llegas a tirar la toalla, te agachas otra vez lo recoges, dices no voy a tirar la toaIla, pues sí, hay momentos donde tiras la toalla, por que no puedes más, o bien por el dolor, o casi siempre ha sido por los efectos secundarios de la quimio, pierdes peso, te debilitas y hay momentos que dices, ostia, he tocado fondo, pero bueno te sirve para coger impulso.

Paco: Muy bien, perfecto, Mercedes, ¿Qué quieres compartir con nosotros?

Mercedes: Bueno, me llamo Mercedes, tengo 59 años, nunca he ido al médico, de repente, hace dos años, piensas por que (llora), y realmente lo estoy llevando muy mal, y si intento llevarlo bien es por mi hija, y eso es lo que me sabe mal, (Ilora) lo siento.

Paco: Sí quiere puedes hablar más tarde ME: Sí, ya hablaré más tarde.

Paco: Cristina,

CRIS: Soy Cristina, tengo 53 años, soy especialista en Publicidad, me diagnosticaron este verano, y todavía estoy en estado de shock.

Paco: Interrumpe para darle un pañuelo a Mercedes. (Continua), perdona Cristina, decía que aún estabas en estado de shock.

CRIS: Sí, todavía estoy en estado de shock, hay momento que pienso que esto no me está pasando a mí, por que no puede ser. Todavía estoy en estado de shock, mi vida era, yo soy una persona muy independiente, me gusta ser independiente, hacer cosas por mi cuenta, sin dar muchas explicaciones, tengo mis gustos, y de golpe todo esto se va a la mierda, por que estoy en casa de mi hermana y mi cuñado, yo vivía sola con mi perrito, muy feliz, yo tampoco había estado enferma, ni siquiera había pasado el sarampión, y entonces pasa esto, y dice esto que es, sabes, ¿pero qué esta pasando?, no puede ser, y todavía estoy en estado de shock, no sé, debería darle la vuelta, debería buscar motivos para vivir, cosas para hacer, yo tengo un larga lista de cosas para hacer, por que yo era una persona muy centrada exclusivamente en mi trabajo, no tenía tiempo, yo decía, bueno, más adelante, cuando tenga un sabático, yo siempre tenía muchas cosas pendientes, ahora tengo mucha pereza, no tengo ganas, a parte de que muchas veces estoy cansada, pero retomando el hilo, ahora vivo tutelada, como si me hubieran atado las manos, me quieren mucho, me cuidan muy bien, estoy bien, pero como pensando, algún día tengo que volver a mi vida de antes, esto es una pesadilla, o sea no me he hecho a la situación, en definitiva, por que pensar así, es que no me he hecho a la situación, estoy en estado de shock todavía. Por ejemplo, no salgo a la calle apenas, no quiero ver a nadie, no es normal, por que la gente se preocupa, pero

Paco: Gracias Cristina, nos hemos presentado cada uno de nosotros, se han expresado experiencias diferentes, expresiones de amenaza e incertidumbre, pero lo importante es expresar, Ilorar, dejarse sentir, por ello os agradezco a todos el haber compartido. Vamos hablar del sentido y del propósito. Hay momento difíciles, hay momento de dificultad de encontrar sentido a nuestra vida, o mantener el sentido de nuestra vida o a veces, la enfermedad nos da una experiencia de crecimiento personal. (A continuación describo los objetivos de cada una de las 8 sesiones del grupo). Vamos a iniciar la primera sesión, hablando de Víctor Frankl, psiquiatra judío, es el autor del libro de la primera tarea para casa, "El hombre en busca del sentido". El tiene una famosa frase, que es la siguiente 
"Quién tiene un porqué para vivir, encontrará casi siempre el cómo". Víctor Frankl fue recluido en un campo de exterminio nazi. Él cuenta cómo a pesar de tener un visado para marcharse sólo él y su mujer de Viena a USA, en los momentos antes de la II guerra mundial, en los momentos de persecución del pueblo judío, decide quedarse. Cuenta como al pasar por una sinagoga destruida, recoge un trozo de la fachada de la sinagoga, y la lleva a su casa, y le pregunta a su padre, a qué corresponde este pequeño trozo de la fachada de la sinagoga. El padre le comenta que es parte de uno de los mandamientos de la religión hebrea "honraras a tu padre y tu madre". Tras escuchar estas palabras decide rechazar el visado, y quedarse en Viena. Más tarde él, su mujer y padres son llevados a los campos de concentración nazi, y sólo sobrevive él. Este libro lo escribe después de la II guerra mundial. Es un libro muy utilizado por psicólogos con el objetivo de ayudar a las personas que por un diagnóstico o por alguna otra razón le restan sentido a su vida. Por eso, os mando por e-mail este libro, para que empecéis a leerlo. Vamos a definir lo que es sentido (se lee la diapositiva cuatro de la sesión $1^{\mathrm{a}}$ de Definiciones de Sentido). La diapositiva que tenéis en la hoja, al lado de la definición de sentido, se refiere a las fuentes del sentido (se lee la diapositiva $3^{a}$ Fuentes de Sentido). Bien, es importante el concepto de sentido y la fuente de sentido se haya quedado clara. ¿Hay alguna duda?. Durante las sesiones iremos haciendo ejercicios. Los ejercicios son preguntas, reflexiones. Antes, ¿Queréis comentar algo de lo que he dicho?. Voy a empezar yo lanzando una pregunta (leo el ejercicio experiencial que figura en la hoja de la primera sesión "Momentos llenos de sentido").

MONT: Yo cuando me casé, al día siguiente de levantarme, tenía libertad, por que en mi casa no me dejaban salir, tenía mi propia casa, era como un pájaro que le abren la jaula y que vuela, yo me sentí en esos momentos así, libre, y esa experiencia recién casada, en mi casa, mi marido, fue extraordinario, y luego la otra, que me ha marcado mucho fue la muerte de mi madre hace 6 años, fue un diagnóstico de cáncer, nos dijeron que era terminal, y no lo he superado todavía, o sea, es que cómo si la tuviera presente, como si la tuviera en los rincones, es una cosa que me ha marcado.

Paco: Gracias Montse, y esas experiencias que tuviste ¿Qué significado, qué sentido tuvo en tu vida, qué te han aportado estas experiencias, en positivo o en negativo?

MONT: A positivo, a positivo, aunque sea negativo que hecho a faltar a mi madre, ha sido positivo, me ha dado más fuerza a la vida.

Paco. ¿Para crecer?

MONT: Para crecer, sí, por que mi madre era una persona negativa, todo lo encontraba mal, a todo le encontraba peros, todo le daba miedo, o sea si no iba con su marido a comprar, no podía ir a comprar sola, o sea, no iba a Barcelona, por que vivíamos en San Just, ella coger un autobús sola a Barcelona, no, no, no, ¿sabes?, yo siento lo contrario, yo he vivido sola, aquí y allá, me ha dado más fuerza, por que he visto el efecto en mi madre, y yo no quiero ser así, quiero ser de otra manera, o sea, que me ha dado más fuerza.

Paco: Gracias Montse. Javier

J: Cosas que te refuerza el sentido de la vida, cuando te vas de viaje, el día a día es muy repetitivo, entonces cuando Ilega las vacaciones, $y$ te puedes ir a un sitio en concreto que te gusta...

Paco: Sobre algo que te ha supuesto a ti

J: Bueno no he tenido ninguna situación estrambótica hasta encontrarme con la enfermedad, entonces ya en el momento de la enfermedad, un momento que te afecta mucho es cuando te juntas con la familia, te apoyan, te juntas con los amigos, te hunde completamente, el forzarte en ello. 
Paco: ¿La amistad es algo que te conmueve, que te da fuerzas, sentido, en estos momentos del diagnóstico?.

J: Sí, en estos momentos el esfuerzo que te supone encontrarte con ellos, recuperar la normalidad.

Paco: Gracias, Cristina.

CRIS: Para mí, el estilo de vida de antes, los momentos más Ilenos de sentido eran los relacionados con el trabajo, por que era la prioridad y también con aprender cosas, también viajando, me ha gustado mucho, y tengo mucha curiosidad con todo, ver cosas, observar y el trabajo, para mi el sentido de mi vida era esto, el trabajo con una doble cara, de dificultad, por cuando vas de viaje y descubres cosas todo es más fácil.

Paco: $\mathrm{Y}$ alguna experiencia negativa.

CRIS: Negativo, hace un año murió mi padre y estuvo varias semanas en coma, y era irreversible la situación, y eso fue muy duro, y para mi fue la experiencia más difícil cercana, o sea, relacionada con la muerte.

Paco: ¿Y qué fuente de sentido le atribuyes a estas experiencias?

CRIS: Según esta clasificación, sería creativas y experienciales.

Paco: Para ti Javier $i$ Tus experiencias serían existenciales?

J: Sí, la amistad..

Paco: Y parta ti Montse, ¿Serían también existenciales?. ¿Cómo legado?.

CRIS: Sí, mi madre, es lo que me ha dado sentido.

Paco: Mercedes, háblanos de una o dos experiencias,

ME: Positivas, las tenidas con las hijas, pero también hay malas, pero bueno, mi trabajo ha sido lo que me ha hundido muchísimo, esto lo que llevo mal, por que antes iba a mi trabajo, y estaba fuera en la calle, trabajando de comercial de Farmacia y ahora

Paco: ¿Siente aceptarlo?

ME: Mucho, y lo intento hacer y no me sale, estas triste, no das lo que tienes que dar, también no lo valoras, te da igual, pues claro,

Paco: ¿Lo valoras ahora más que antes?

CRIS: Valoro ahora no hacerlo, antes no lo valoraba, antes pensaba que era mi trabajo, y ahora valoro no hacerlo.

Paco: El hecho de no hacerlo.

ME: Claro, y es una pena, ¿Tiene sentido la vida?, si no haces nada,

Paco: Pero si decías que tenía sentido la vida por tus hijas.

ME: Sí, pero yo no puedo estar encima de mis hijas, mis hijas tienen su vida, tampoco las quiero agobiar. Yo no puedo decirle a mis hijas ayudarme, no, soy yo la que tengo que ayudar. Sí, yo no veo nada, no es si vas a durar más o menos. La única meta que tengo es llegar al 28 de mayo, que es cuando mi hija se casa. Lo demás es que tampoco, tampoco te dicen si vas a durar esto, lo otro, lo otro, no te dan, no te valoran el tiempo que vas a estar tu con esta enfermedad. Yo antes era una persona muy activa, estaba todo el día trabajando, y ahora es que, no hago nada. Yo antes me reía, me reía, saltaba, bailaba, cantaba, y ahora nada, se me ha ido toda la expresión de la cara. Pero no veo sentido a nada, con eso no quiero decir que me quiera morir, tampoco quiero sufrir, pero pienso que a lo mejor, no sé, si hubiera algo más positivo, pues es que tampoco, lo pasas fatal con la quimio, si uno tuviera paciencia, pero es que, cada día te levantas igual, cada día lo mismo, igual te da esto lo otro, antes iba superarreglada, y ahora, mi pelo iba siendo rubia y ahora no puedo ponerlo rubio, y todo esto me (silencio)

Paco: Para mí tiene mucho valor lo que tú estás haciendo.

ME: No, yo creo que no, creo me estoy malformando, no sé, mis hijas me dicen, amablemente "cambia la cara". Eso es que no puedes. En realidad lo pasas fatal, ni un día, estaba en la mesa, y es que como si no estuvieras, y alguno podría pensar, si 
a lo mejor el año que vienes ya no estas, tampoco lo valoran.

Paco: Pero lo pensabas.

ME: Como futuro no veo, pero tampoco veo que te digan, tranquila, pero tampoco te pueden decir vas a durar una año, dos o tres, pero es que no

Paco: Cuando has oído a Javier, Cristina y a Montse, ¿Qué sensaciones has tenido, cuando hablaban de lo que les daba sentido?.

ME: Pues que ahora no tengo mucho sentido, mucha fuerza, me gustaría cambiar, y pensar que lo tengo, pero ha venido y saberlo llevar, pero no pasa nada, hay mucha gente con

Paco: ¿Qué crees que necesitas?

ME: Creo que necesito tener más contactos con gente que tengan esa experiencia y que sepan ayudarme, a través de la experiencia de los otros, y también como tú puedes intentar ayudarles

Paco: Gracias. Maribel o yo no te podemos decir que te entendemos, te pueden entender otras personas que están en la misma situación. Esta es la razón por la cual estamos todos aquí reunidos. ¿Queréis comentarle algo a Mercedes?

MONT: Yo me sentía así al principio, me daba igual todo, no me dijeron ni un tiempo, ni nada, me dijeron que era un tumor, me sacaron un riñón, y al mes se formó otro tumor en el mismo sitio, y me dijeron que era imposible, que me lo habían limpiado, pero si es imposible, ¿Por qué me ha vuelto a salir?. Era como si estuvieras en una nube, en un choque, y dices, a ver, esto no puede estar pasándome a mí, y me daba igual todo, y te paras a pensar, y dices, no, no, tengo que luchar como sea. Yo era rubia platino, el pelo hasta aquí, y lo primero que hice fue cortármelo corto, y buscando gorros. Pero mi marido me dijo. ¿Qué haces?. En casa, algún gorro, alguna boina. Por que yo ya estaba desesperada. Yo era peluquera, tenía una peluquería, mi imagen era lo primero, yo me reía de todo, supersimpática, sabes, y de porrazo, tengo mala leche, ahora la tengo, todavía la tengo la mala leche, cuando me encuentro mal, soy insoportable, o sea, cuando me duele todo, incluso me molesta incluso mis hijas, los gritos de ella, le digo a mi marido, Ilévatelas, Ilévalas al parque, quiero estar sola, o leyendo un libro, o viendo la tele, pues claro, ellas viendo todo el día dibujos, no se, aunque sea una película, de hacerme llorar, o lo que sea, es igual. Lo que quiero es estar sola. Ya ahora se, con el tiempo, necesitas tiempo, por que yo hace ya un año y medio que estoy

ME: Es que yo llevo 4 meses, llevo desde Octubre. No llevo tanto.

MONT: Yo Ilevo año y medio

JA: Yo llevo dos años. Yo quiero comentar, que a pesar que pones todo lo de ti mismo, a principio no, y segundo, esto que te pasa a ti con tus hijas, me pasa a mi con las mías. A veces te molesta, por que cuando estas con dolor, te molesta la moto que pasa, te molesta todo, a pesar que intento ser optimista con ésto, y llevarlo de la mejor manera, no puedes ir "happy, happy" todo el día. Qué vamos hacer.

Paco: Hablamos José Antonio de esa preguntas, una o dos experiencias.

$J A$ : Yo no sé, si ubicarla como una experiencia que le ha dado sentido, si que una experiencia que me impacto mucho, y además fue aquí, fue saliendo de aquí, de la consulta del oncólogo y esperando ahí, en el mostrador de ahí fuera, para programar, había un chico de mi edad, un poco desaliñado de aspecto, pero estaba ahí esperando, y bueno no presté atención, pero entró una especie de camillero, celador, supongo que venía a buscarlo para llevarlo, y esto que le preguntó, puede parecerte absurdo, ¿Qué pasa, como va eso?, y le dijo para lo que nos queda en el convento. O sea, aquello me impactó muchísimo, no lo que dijo, sino cómo lo dijo, la cara que puso. $\mathrm{Y}$ entonces en aquel momento, me dije, "ostia soy un tío afortunado", o sea 
me sentí el tío más afortunado del mundo, por que aquella persona, yo la vi sola. La vi completamente sola. Pero no allí, sola en su mundo, en su vida. Yo tengo la suerte, que a mí me llama los amigos, para mí es importantísimo los amigos, es importante dejarse visitar, dejarse ayudar. Por lo menos para mí, me reconforta mucho, la familia, yo nunca he venido aquí solo. Vengo con mi mujer, vengo con mis amigos, con primos, siempre. Siempre me llaman, o me visitan, y se que están allí. Compañeros de trabajo, para mí están siendo un apoyo fundamental. Y creo que soy afortunado en ese aspecto. Pero era lo que decía, en aquel momento me impactó muchísimo, y desde entonces me siento bien, en el aspecto ese, de que estoy superarropado, y sé que estoy superarropado, y a principio también, hasta hace poco estaba pasando esto de que no quiero que la gente se esfuerce, se preocupe, no quiero preocupar a los demás

CRIS: Sí, me das pena que tengas pena. Yo me arreglo, boina, gorros, colección tengo. Eso es lo que primero hice, me hizo sentirme bien. Pero la cuestión, a mí me da pena darles pena.

JA: Tú decías que estabas en casa de tu hermana.

$C R I S: \mathrm{Si}$, en casa de mi hermana.

$J A$ : Imagina que fuera al revés, tu hermana se encuentra en la situación que tu estas, ¿Tu que harías? , darle todo el amor del mundo, y no por que te daría pena, sino por que necesitas darle ese apoyo, por eres su hermana, y te encuentras con esa necesidad. Eso fue lo que me dijeron a mí, la fiestas, cuantas más gente, más fiestas son, y la espera, cuanto más apoyo, menos espera son.

MONT: Más llevaderas.

JA: Más llevaderas. Yo creo que es así. Y yo te digo, hasta hace muy poco, soy muy de no molestar, por que la gente lo hace por corazón, por que quieren. No por que le des pena. Habrá gente que si, el vecino que piensa, el pobre de éste, que le queda dos telediarios. La gente cercana a ti, la gente que realmente te quiere, para nada.

MONT: La verdad, cuando pasas una enfermedad como ésta, te das cuenta la gente que realmente te quiere, y la que no. La que dices, ostia, ¿esta es amiga mía?. Y no me ha llamado, y se ha enterado que tengo cáncer, y no me ha llamado. Te das cuenta la gente que tienes a tu alrededor. Te das cuenta de la gente que te quiere, que te está llamando, que está apoyando, que si necesitas cualquier cosa, que viene a casa, que te puedes tomar un café, y que tienes que abrirle la puerta, por que lo ves sincero, lo ves tan, tan, que tienes que abrirle la puerta. Yo al principio, también pensaba, ostia es que las primeras semana no se lo contaba a nadie, sólo a la familia íntima. Y entonces a las amistades, cuando yo estuve preparada para contar lo que me pasaba, tarde 15 días. ¡Eh!, por que no quería ver a nadie. Decían ¿Cómo es que no viene Montse por el cole?. Entonces le decían es que tienen mucho trabajo, no sé qué, no sé cuántos, yo no quería ver a nadie, hasta que pensé voy a contar poco a poco, y entonces te das cuenta de la gente, yo me siento supearropada. El día que mi marido estaba trabajando, yo me iba a casa de mi suegra, no puedo cargar con las niñas. Nos vamos todos a casa de mi suegra, y el fin de semana a casa con mi marido y mis hijas, y lo sabía mi suegra, y la mujer tiene 75 años. Y ella tiene una edad que no está para estos trotes, pero por el momento ella está fuerte y está bien, pues me siento supearropada, por la familia, por los amigos.

CRIS: En mi caso, es curioso yo vivo en un pueblo, y entonces todo el mundo se ha enterado. Mi hermana vive a las fueras, que no ves a nadie, yo saco a mi perro, yo encantada, no me va nadie, no veo a nadie. Entonces que pasa, que cuando mi hermana, mi madre salen, me pasan el parte. Oye recuerdos de tal, de tal, de tal. Oye yo no lo dicho, pero la gente se lo dicen entre ellos. La gente la conocemos, 
y dicen la hija de tal, ¿Sabe lo que le ha pasado?. Y es un poco gracioso.

MONT: Yo nací en un pueblo pequeño, y hasta el cura se enteró. Por que tenemos amistad, o sea que, se entero toda la iglesia.

CRIS: En mi caso igual.

MONT: ¿Qué te quería decir?, que gente que me lo esperaba, que vinieran y se preocuparan. O sea que es de agradecer.

CRIS: Así que empezaré a contestar, por que no contesto.

MONT: A mi me pasó, los primeros 15 días.

Paco: Gracias. Luisa, ¿alguna experiencia personal que quieras comentar?.

$L U$ : Pues yo, una experiencia fue cuando me dijeron lo que tenía, yo lo sabía. Yo lo iba asimilando, entonces se lo dije a mi familia, que no viven aquí, solo estamos mi hija y yo, pues me preguntaron como lo estaba llevando, que una sobrina iba muy seria por la calle, le preguntaban, que si había hecho una comida familiar, y todo estaban triste, y le preguntaban porque. Cuando me dijeron eso, pensé que yo no puedo hacer sufrir a mi familia, yo tengo que ayudarme, por eso empecé a llamarles, a decirles que estaba bien, y luego en el hospital el apoyo que tuve. Después de Bellvitge vine aquí, todos los dolores se me quitaron pero no podía ponerme de pié. El desánimo me ha venido después. Yo fui asimilando poco a poco. Pensé a lo mejor me equivoco. Yo me ingresé por dolores, y como me seguían, cuando yo miré el TAC, y salí una frase de "tumor", ponía otra frase: "metástasis", y no vuelto a mirar nada más. Claro, cuando ingresé, ya sabía lo que tenía.

Paco: Habéis dado un testimonio de experiencias importantes, con sentido. Tengo que deciros un para de cosas, primero, que yo me comprometo, junto con Maribel a estar aquí reunidos con todos vosotros, y os pido vuestro compromiso de venir a la sesiones de grupo. Habrá sesiones que aporten y otras que nos aporten nada. El hecho de venir y compartir, es lo que más ayuda. Por eso, os pido el compromiso de que vengáis a las sesiones de grupo. Y lo segundo, es que os enviaré un libro por mail, el libro de Víctor Frankl, "El hombre en busca del sentido", para que lo empecéis a leer, y lo comentamos la próxima sesión. Antes de acabar, no se si queréis comentar o alguna pregunta. Pues nos vemos la próxima sesión. Gracias por venir.

\section{EVIDENCIA SOBRE LA EFICACIA}

Los resultados de los grupos de psicoterapia centrada en el sentido de Breitbart et al. ${ }^{(5)}$ y Gil (estudio en curso) muestran una reducción de la sintomatología ansiosa y depresiva, desesperanza y deseo de adelantar la muerte en pacientes con cáncer avanzado, a la vez de aumentar el bienestar espiritual y la sensación de sentido $^{(14,15,16)}$ (ver tabla 1 y tabla 2). Asimismo, como se observa en la figura 1 , el sentido, la sensación de paz y la fe se ve aumentada tras la intervención de grupo.

\section{Tabla 1. Resultados Pre y Post Intervención (finalizada la octava sesión de grupo) $n=37$. Breitbart et al. (5)}

\begin{tabular}{|l|c|c|}
\hline Pre- Intervención & Post-Interv. & P. \\
\hline Desesperanza $=6,76$ & 5,81 & 0,070 \\
\hline Depresión $=14,73$ & 15,35 & 0,090 \\
\hline Ansiedad $=2,29$ & 2,16 & 0,100 \\
\hline FACIT $($ Sentido/Paz/Fe) $=2,06$ & 2,53 & 0,000 \\
\hline
\end{tabular}


Tabla 2. Resultados Pre-Post Intervención (finalizada la octava sesión de grupo) n=6. Gil, 2013 (Estudio en curso)

\begin{tabular}{|l|c|c|}
\hline Pre- Intervención & Post-Interv. & P. \\
\hline Desesperanza $=5,08$ & 4,57 & 0,001 \\
\hline Depresión $=7,23$ & 6,71 & 0,003 \\
\hline Ansiedad $=9,62$ & 7,57 & 0,001 \\
\hline FACIT $($ Sentido/Paz/Fe) $=2,35$ & 2,48 & 0,000 \\
\hline
\end{tabular}

Figura 1. Escala Española de Bienestar Espiritual (FACIT-Sp-12). Puntuación Total: Subescalas Sentido/Paz y Fe.

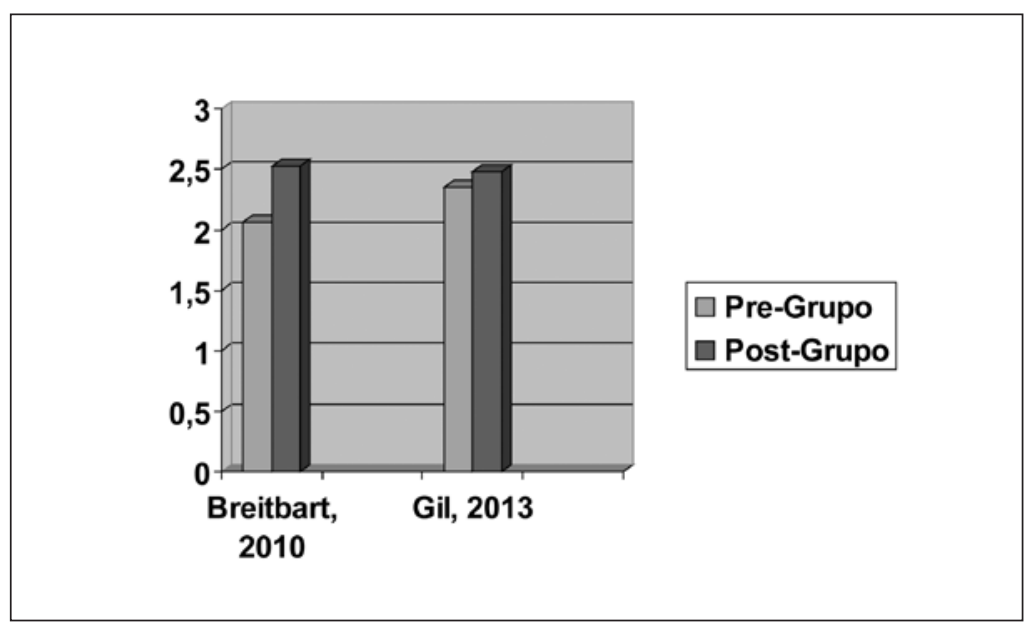

En relación a los resultados del estudio piloto realizado por Gil en el Hospital Duran i Reynals, Instituto Catalán de Oncología, podemos concluir lo siguiente:

1. Hemos mejorado el manual del terapeuta del presente modelo de psicoterapia, adaptando los ejercicios experienciales y las tareas entre las sesiones para nuestros pacientes con cáncer.

2. Ante la pérdida de pacientes en la asistencia a las sesiones, principalmente por problemas de salud (toxicidad a la quimioterapia, o aumento de sintomatología) para el siguiente proceso de validación, una vez acabado el estudio piloto, recomendamos: a. Aumentar el número de reclutamiento de pacientes al inicio de la terapia a 10-12 pacientes, ya que estimamos una pérdida de 4 a 5 pacientes a lo largo de las sesiones de grupo.

b. Ampliar el tiempo de supervivencia de los pacientes participantes del año a los 56 meses.

c. Incluir pacientes en estadios II, III y IV.

d. Facilitar la participación de pacientes con enfermedad avanzada desde su domicilio a través de dispositivos online. 


\section{RESUMEN}

Estos resultados logrados sugieren la necesidad de desarrollar intervenciones psicoterapéuticas para ayudar a los pacientes con cáncer a mantener y aumentar su sensación de sentido y propósito de vida a pesar de su enfermedad.

\section{REFERENCIAS BIBLIOGRÁFICAS}

1. Gil F, Costa G; Pérez X, Salamero M, Sánchez N, Sirgo A. Adaptación psicológica y prevalencia de trastornos mentales en cáncer. Med Clin 2008;130:90-2.

2. Gil F, Costa G, Pérez J. Does chemotherapy reduce stress?. Palliat Support Care 2010;8:455-60. Doi: 10.1017/ S1478951510000337

3. Gil F, Costa G, Hilker I, Benito L. First anxiety, afterwards depression: psychological distress in cancer patients at the diagnosis and after the medical treatment. Stress Health 2012;28:362-7. Doi: 10.1002/smi.2445

4. Breitbart W, Poppito S, Rosenfeld B, Vickers AJ, Li Y, Abbey J, Olden $M$ et al. Pilot randomized controlled trial of individual Meaning-Centered Psychotherapy for patients with advanced cancer. J Clin Oncol 2012;30:1304-9. Doi: 10.1200/ JCO.2011.36.2517

5. Breitbart W, Rosenfeld B, Gibson C, Pessin H, Poppito S, Nelson C, et al. Meaningcentered group psychotherapy for patients with advanced cancer: a pilot randomized controlled trial. Psychooncology 2010;19:21-8. Doi: 10.1002/pon.1556

6. Rosenfeld B, Pessin H, Lewis C, Abbey J, Olden M, Brescia R. Assessing hopelessness in terminally ill cancer patients: development of the hopelessness assessment in illness questionnaire. Psychol Assess 2011; 23:325-36. Doi: 10.1037/a0021767

7. Peterman AH, Fitchett G, Brady MJ, Hernandez L, Cella D. Measuring spiritual well-being in people with cancer: the functional assessment of chronic illness
therapy-Spiritual Well-being Scale (FACITSp). Ann Behav Med 2002;24:49-58. Doi: 10.1207/S15324796ABM2401_06

8. Scheier MF, Carver CS, Bridges MW. Distinguishing optimism from neuroticism (and trait anxiety, self-mastery, and self-esteem): A re-evaluation of the Life Orientation Test. J Pers Social Psychol 1985;67,1063-78.

9. Zigmond AS, Snaith RP. The Hospital Anxiety and Depression Scale. Acta Psychiatr Scand 1983,67:361-70. Doi: 10.1111/j.1600-0447.1983.tb09716.x

10. Costa G, Pérez X, Salamero M, Gil F. Discriminación del malestar emocional en pacientes oncológicos utilizando la escala de ansiedad y depresión hospitalaria (HADS). Ansiedad Estrés 2009;15:217-29.

11. Rosenfeld B, Breitbart W, Galietta M, Kaim M, Funesti-Esch J, Pessin H, Nelson CJ, Brescia R. The schedule of attitudes toward hastened death. Cancer 2000;15:2868-75. Doi: 10.1002/1097-0142(20000615)88:1 $2<2868:: A I D-C N C R 30>3.0 . C O ; 2-K$

12. Costa G, Gil F. Crecimiento postraumático en pacientes oncológicos. Análisis y Mod Cond 2007;33:229-50.

13. Passik S, Inman A, Kirsh K, Theobald D, Dickerson P. Initial validation of a scale to measure purposelessness, understimulation, and boredom in cancer patients: Toward a redefinition of depression in advanced disease. Palliat Support Care 2003;1:41-50. Doi: $10.1017 \% 2 F S 1478951503030062$

14. Breitbart W, Rosenfeld B, Pessin H, Kaim M, Funesti-Esch J, Galietta M, et al. Depression, hopelessness and desire for hastened death in terminally ill patients with cancer. J Am Med Assoc 2000;284:290711. Doi:10.1001/jama.284.22.2907.

15. McClain C, Rosenfeld B, Breitbart W. The influence of spirituality on end-of-life despair among terminally ill cancer patients. Lancet 2003;361:1603-7. Doi:10.1016/ S0140-6736(03)13310-7

16. Nelson C, Rosenfeld B, Breitbart W, Galietta M. Spirituality, depression and religion in the terminally ill. Psychosomatics 2002;43:21320. Doi: 10.1176/appi.psy.43.3.213 
